\title{
Genome-Wide Analysis of the Thioredoxin Gene Family in Gossypium hirsutum L. and the Role of the Atypical Thioredoxin Gene GhTRXL3-2 in Flowering
}

\author{
Hui Liu ${ }^{1} \cdot$ Yunfei $\mathrm{Li}^{1} \cdot$ Xianzhong Huang ${ }^{2,3}$ (])
}

Received: 10 February 2021 / Revised: 14 April 2021 / Accepted: 22 April 2021 / Published online: 7 May 2021

(c) The Author(s) 2021

\begin{abstract}
Thioredoxin (TRX) is a highly conserved low-molecular-weight protein and a ubiquitous antioxidant enzyme that plays key role in the regulation of plant growth and development. Here, using the whole-genome sequence, we performed a systematic analysis for the TRX gene family in upland cotton (Gossypium hirsutum L.) and analyzed their structural characteristics, evolution, and expression profiles during growth and development. At least 86 GhTRX members, 40 typical and 46 atypical, were identified in the cotton genome, and they were unevenly distributed on the 26 chromosomes. Conserved domains and phylogenic tree construction classified the typical TRX gene family into seven subfamilies and the atypical TRX into nine subfamilies. An evolutionary analysis revealed that the TRX gene family underwent purification selection during evolution. In addition, an RNA-Seq analysis showed that, during vegetative and reproductive development, the differences in transcript abundance levels and organ-specific expression patterns suggest functional diversity. Biochemical assays demonstrated that the atypical TRX protein GhTRXL3-2 interacted with the cotton FLOWERING LOCUS T protein GhFT. The overexpression of GhTRXL3-2 in Arabidopsis thaliana resulted in early flowering compared with control plants. Additionally, the silencing of GhTRXL3-2 in cotton delayed maturation, suggesting that it has important roles in cotton's flowering regulation. These results help clarify the evolution of the TRX genes and elucidate their biological functions in cotton flowering regulation.
\end{abstract}

Keywords Thioredoxin (TRX) $\cdot$ Cotton $($ Gossypium hirsutum L.) $\cdot$ Florigen $\cdot$ Flowering $\cdot$ Gene expression

\section{Introduction}

Thioredoxin (TRX) is a kind of small redox protein that contains the conserved WC(G/P)PC motif, which catalyzes the exchange of sulfhydryl disulfide bonds, and the protein plays significant roles in maintaining cellular redox homeostasis (Meyer et al. 2009; Heide et al. 2004). In plants, TRX regulates a series of key functions, such as photosynthesis, flowering, seed development and germination, and intercellular

Xianzhong Huang

huangxz@ahstu.edu.cn

1 College of Life Sciences, Shihezi University, No. 221 North 4 Road, Shihezi 832003, China

2 Center for Crop Biotechnology, College of Agriculture, Anhui Science and Technology University, No. 9 Donghua Road, Fengyang 233100, China

3 Special Plant Genomics Laboratory, College of Life Sciences, Shihezi University, No. 221 North 4 Road, Shihezi 832003, China communication (Potters et al. 2010). Despite the existence of many TRX protein variants in plants, the two cysteine (Cys-X-X-Cys) residues at the active site provide sulfhydryl (SH) groups, which are involved in the reducing activity. The reduced form of TRX, free TRX-(SH) ${ }_{2}$, reduces the bonds of disulfide target proteins, while the oxidized form, TRX-S $\mathrm{S}_{2}$, contains a disulfide bond in the active site. It is reduced to TRX-(SH) $)_{2}$ dithiol by NADPH and TRX reductases (Meyer et al. 2008).

The TRX family members are divided into two categories, typical and atypical (Chibani et al., 2012). On the basis of the similarity levels of the main amino acid, the molecular functions, and the localization within a cell, the typical TRXs include the H, F, M, X, Y, Z, and O subfamilies. Most of the typical TRXs contain a canonical WCGPC active site, and are generally reduced by TRX reductases (Gelhaye et al. 2005; Meyer et al. 2012; Delorme-Hinoux et al. 2016). The atypical TRX forms the TRX-like subfamily and its members contain the WCRKC and WCRVC active sites (Cain et al. 2009; Chibani et al. 2012; Dangoor et al. 2009). TRX 
proteins are widely distributed in subcellular locations including the nucleus, apoplasts, extracellular matrices, and phloem saps, and the TRXs are involved in many different cellular processes, including protein assembly and folding, translation, hormone synthesis, plant development seed germination, oxidative stress responses, and intercellular communication. Moreover, the recent sequencing of many plant genomes has led to the identification of more TRX proteins (Clot, Trx-like 1 and -2, and Trx-lilium1, -2, and -3) that contain atypical active site sequences having varied amino acids between the conserved cysteines, so these TRXs were classified as atypical TRX proteins (Chibani et al. 2012; Delorme-Hinoux et al. 2016). They possess different active sites, such as WCPDC (Clot), WCRVC or WCRKC (TRXlike proteins), GCGGC, SCGSC, or WCASC (Trx-lilium) (Chibani et al. 2012; Meyer et al. 2012). However, little functional information is available on these atypical TRXs.

In plants, flowering is not only a physiological phenomenon, but also an important agronomical trait. It is closely correlated with the environmental adaptability of plants and crop yield. The FLOWERING LOCUS T (FT) protein, the main component of florigen, is a critical integrator of endogenous developmental cues and external environmental conditions (Fornara et al. 2010). The rice HEADING DATE3 protein, a homolog of FT, is transported from leaf phloem to the apical cells of shoots through vascular tissues. It interacts with the intracellular receptor protein 14-3-3 and then binds bZIP transcription factor FD1 to yield the florigen activation complex (FAC). The resulting FAC activates OsMADS15, an APETALAl homolog, which leads to flowering induction (Taoka et al. 2011).

Cotton is the most important fiber crop worldwide, and early maturity is an important goal of most cotton breeding programs. A homolog of FT from G. hirsutum, GhFT, was characterized in the previous studies (Guo et al. 2015; $\mathrm{Li}$ et al. 2015). We have investigated the molecular nature of cotton FAC homologs and identified an interaction protein with GhFT using a yeast-two-hybrid (Y2H) screening experiment that was encoded by $G h \_A 05 G 2534$ gene (data not shown). An amino acid alignment showed that Gh_A05G2534 encodes a TRX-like 3-2 protein, belonging to the atypical TRX family member. This gene was designated GhTRXL3-2. These results prompted us to characterize the TRX members in cotton. To date, limited molecular and functional information is available on the TRX family members in cotton. The successful sequencing and the recent assembly of the G. hirsutum genome enable the more convenient and reliable characterization of a gene family $(\mathrm{Hu}$ et al. 2019; Huang et al. 2020).

In this study, we aimed to identify the $T R X$ genes from cotton genomes and to classify the TRX proteins using domain and protein sequences, gene structures, phylogenetic relationships, synteny, and gene expression profiles from different tissues. Additionally, we cloned the GhTRXL3-2 gene from the upland cotton genome, and explored its functions by virus-induced gene silencing (VIGS) and heterologous overexpression in Arabidopsis thaliana. These results lay the foundation for further studies on the functional genomics of the TRX gene family in cotton.

\section{Materials and Methods}

\section{Plant Materials}

Cotton seeds (G. hirsutum L. cv. XLZ 33) were field-grown under natural conditions during the summer of every year in Xinjiang, the Northwestern cotton-growing region of China, Shihezi. For tissue expression analysis, roots, stems, leaves, and shoot apical meristems (SAMs) were collected at the third true-leaf expanding stage. Fiber-bearing ovules were harvested at the following time-points during development: $-3,0$, and 5 day post-anthesis (DPA). Fibers were sampled at 10 and 20 DPA. Petals were collected at flowering stage. All samples were immediately frozen in liquid nitrogen and stored at $-80^{\circ} \mathrm{C}$ until RNA extraction.

The seeds of $A$. thaliana Columbia (Col-0) were surface sterilized as previously described (Guo et al. 2015). The sterilized seeds were stratified for 3 days at $4{ }^{\circ} \mathrm{C}$ in darkness and then plated in Petri dishes with half-strength Murashige-Skoog salt mixture (Duchefa, Haarlem, The Netherlands), pH 5.8. After 10 days, the Arabidopsis seedlings were planted in pots containing soil:vermiculite:perlite (2:1:1) and placed in a growth chamber under long-day (LD) conditions (16-h light/8-h dark, $23 / 20{ }^{\circ} \mathrm{C}$ day/light, $200 \mu \mathrm{mol} \mathrm{m}{ }^{-2} \mathrm{~s}^{-1}$ ).

\section{Identification and Structural Characterization of the TRX Genes in Gossypium hirsutum}

Putative cotton TRX members were identified using a BLAST search against the upland cotton standard line 'TM-1' genome database (v1.1) (Zhang et al. 2015) using Arabidopsis TRX protein sequences as queries with an $E$ value of $1 \times 10^{-10}$. The TM-1 gene annotation results were downloaded from CottonGen (https://www.cottongen.org/). All the retrieved putative proteins were further submitted to PFAM (http://pfam.xfam.org/), SMART (http://smart. embl-heidelberg.de/smart/batch.pl), and InterProScan (http://www.ebi.ac.uk/interpro/) for annotation of domain structures. The molecular weight and theoretical isoelectric point of each GhTRX protein were analyzed using the online ExPASy tool (https://www.expasy.org/). Predictions of transmembrane helices were performed using the online TMHMM Server v.2.0 program (http://www.cbs.dtu.dk/servi ces/TMHMM/). The online SignalP-3.0 Server tool (http:// 
www.cbs.dtu.dk/services/SignalP-3.0/) was used to predict the presence and location of signal peptide cleavage sites in the amino acid sequences of GhTRX proteins. The online WoLF PSORT tool (https://wolfpsort.hgc.jp/) was used to predict the subcellular localizations of the GhTRX proteins. Intron-exon structure was determined by aligning genomic DNA and full-length cDNA sequences using Gene Structure Display Server 2.0 (http://gsds.cbi.pku.edu.cn/) (Hu et al. 2015).

\section{Phylogenetic Analysis and Conserved Motif Distributions}

Multiple amino acid alignments were performed using CLUSTAL_W and the phylogenetic tree was constructed using the neighbour-joining algorithm with a bootstrap value of 1000 in the MEGA 7.0 (Kumar et al. 2016). Conserved motifs were determined using the online tool Multiple Em for Motif Elicitation (version 5.3.0) (http://meme-suite.org/ tools/meme) (Bailey et al. 2015) with default parameters.

\section{Chromosomal Distributions, Collinearity, and Selection Pressure Analyses of the GhTRX Family}

The locational information for GhTRX genes was extracted from the G. hirsutum gene annotation v1.1 (Zhang et al. 2015), and these candidate GhTRX genes were mapped the corresponding chromosomes using the software MapInspect (http://mapinspect.software.informer.com/).

In the collinearity analysis, MCScanX (Wang et al. 2012) was used to predict the homologous genes within the species with the default parameters. Molecular evolutionary analyses of the GhTRX genes were performed by calculating the nonsynonymous $(\mathrm{Ka})$ to synonymous $(\mathrm{Ks})$ substitution rate ratios using the KaKs_Calculator online Calculator in TBtools (https://github.com/CJ-Chen/TBtools) (Chen et al. 2020). The results of collinearity analyses within GhTRX genes were visualized using Circos software (Krzywinski et al. 2009).

\section{RNA Extraction and qRT-PCR Analyses}

Total RNA was extracted from cotton tissues collected using the EASYspin Plus Plant RNA Rapid Extraction Kit (Aidlab Biotech, Beijing, China) and was then treated with DNAfree DNase (Invitrogen, Carlsbad, CA, USA). For reverse transcription, M-MLV Reverse Transcriptase Kit (Bioteke Corporation, Beijing, China) was used to synthesize the first strand of cDNA following the manufacturer's instructions. qRT-PCR was performed with the Applied Biosystems 7500 Fast Real-Time PCR System (Life Technologies, Foster City, CA, USA) with the Fast SYBR Mixture (with ROX) (CWBIO, Beijing, China). GhUBQ7 (DQ1 16441) was used as an internal reference gene (Shi et al. 2006). The qRT-PCR primers are listed in Supplementary file 2. All qRT-PCR assays were performed using three independent biological replicates, each with three technical replicates. The PCR conditions and the calculation method of relative gene expression were the same as before ( $\mathrm{Si}$ et al. 2018).

The RNA-Seq data of different $G$. hirsutum tissues were downloaded from PRJNA490626 (Hu et al. 2019). Eight different tissues, roots, stems, leaves, toruses, sepals, petals, pistils, and filaments, as well as ovules from - 3, 0, 1, 3 , and 5 DPA and fibers from 10, 15, 20, and 25 DPA were selected for the expression analysis. Subsequently, the fragments per kilobase of exon model per million mapped reads (FPKM) values were used to measure the gene expression level of each gene with Cufflinks (Version 2.2.2) (http://cuffl inks.cbcb.umd.edu/) (Trapnell et al. 2012). The expression heatmap was manufactured using R software with the logtransformed FPKM $(\log 2[\mathrm{FPKM}])$ values.

\section{Yeast Two-Hybrid (Y2H)}

The $\mathrm{Y} 2 \mathrm{H}$ assays were performed as previously described ( $\mathrm{Si}$ et al. 2018). The full-length CDS of GhFT was separately cloned into a pGBKT7-GAL4 vector (Clontech, Mountain View, CA, USA) as bait plasmids. The full-length CDS of each GhTRXL3-2 was cloned into pGADT7 as the prey constructs. The resulting prey and bait constructs were cotransformed into the yeast strain AH109 as per the description of $\mathrm{Si}$ et al. 2018. The transformants were grown on SD/-Leu/Trp medium and then transferred to SD/-Leu/-Trp/-His-Ade medium with $3 \mathrm{mM} 3$-AT to detect the interaction.

\section{Split Firefly Luciferase Complementation (SFLC) Assays}

For the bimolecular luciferase complementation assays, coding regions of GhTRXL3-2 and GhFT were cloned into pDnor221 by BP reactions. An entry clone of GhFT was introduced into a $35 \mathrm{~S}:$ NLuc plasmid to generate 35S:GhFT-NLuc fusion construct by LR reaction, and entry clone of GhTRXL3-2 was introduced into 35S:CLuc plasmid by LR reactions to generate 35S:CLuc-GhTRXL3-2 fusion construct. 35S:NLuc, 35S:CLuc, and the expression constructs were mobilized into A. tumefaciens GV3101 for $N$. benthamiana transformation. The methods of transiently transformation and observation were the same as $\mathrm{Si}$ et al. (2018). An in vivo plant imaging system (LB 985 NightSHADE, Berthold Technologies, Bad Wildbad, Germany) was used to capture the LUC image. There were at least three biological replicates with independent plants for each assay. 


\section{Gene Cloning and Transgenic Plant Construction}

The full-length coding region of the GhTRXL3-2 gene was amplified by PCR using gene-specific primers (Supplementary file 2), sub-cloned into the pMD-19 carrier (TaKaRa, Dalian, China), and sequenced. The positive plasmid was transferred to the binary vector pCAMBIA 2300-35S-OCS to generate $35 S: G h T R X L 3-2$, which was then introduced into Agrobacterium tumefaciens GV3101.

Transgenic plants were generated using the floral dip method (Clough and Bent 1998), and the homozygous 35S:GhTRXL3 transgenic plants were screened as described by $\mathrm{Si}$ et al. (2018). For qRT-PCR analyses, the rosette leaves were collected from 14-day-old Arabidopsis Col-0 and 35S:GhTRXL3-2 transgenic plants at 11:00 am during the day. The methods were described above. The qRT-PCR primers were listed in Supplementary file 2. Phenotypic observations and statistical analyses were performed as previously described (Guo et al. 2015; Si et al. 2018). To measure flowering time, one homozygous transgenic plant was planted in a pot containing soil:vermiculite:perlite $(2: 1: 1)$.

\section{VIGS Assays}

Seeds of the G. hirsutum var. XLZ 33 were grown under controlled environmental conditions at $22{ }^{\circ} \mathrm{C}$ under LD conditions. For the VIGS assays, a 320-bp fragment of GhTRXL3-2 was cloned by PCR and inserted separately into EcoRI and KpnI sites of pTRV2 vectors to generate the pTRV:GhTRXL3-2 construct using gene-specific primers (Supplementary Table S1). A 420-bp fragment of the cotton CLOROPLASTOS ALTERADOS 1 (CLA1) gene was amplified to generate $p T R V: G h C L A l$ control, which was used as a control (Gao et al. 2011). The transformation and cultivation of Agrobacterium strain GV3101, and the VIGS assays, were performed as previously described (Gao et al. 2011; Si et al. 2018).

\section{Results}

\section{Genome-Wide Identification of TRX Family Members in G. hirsutum L.}

A genome-wide survey identified 86 putative $T R X$ genes from $G$. hirsutum according to the 'TM-1' genome atlas (Zhang et al. 2015). They encoded 40 typical and 46 atypical TRX proteins on the basis of their domain compositions. The biochemical properties of the GhTRX family proteins are listed in Supplementary files 3 and 4. The GhTRX family members encoded sequences of 65-595 amino acids, with an average length of 212 amino acids. Accordingly, the molecular weights varied from 7.56 to $65.81 \mathrm{kDa}$, and the theoretical isoelectric points ranged from 4.45 to 8.60 . Of the 86 GhTRX family members, TRXs having a transmembrane structure accounted for $3.5 \%$, and TRXs having a signal peptide accounted for $8.1 \%$. In addition, a subcellular location analysis revealed that 56, 11, and 10 GhTRX proteins localized to the chloroplast, cytoplasm, and nuclei, respectively. Some GhTRX proteins localized to the endoplasmic reticulum and the mitochondria. The molecular structures and subcellular localization characteristics of the GhTRXs indicated their functional diversity.

\section{Phylogenetic Analysis and Conserved Motif of GhTRX Family Members}

The phylogenetic relationships and domain identifications that were based on the Pfam results revealed that the 40 typical GhTRX proteins were divided into seven subfamilies, H, O, F, Z, X, Y, and M (Fig. 1a). Of the seven subfamilies, the $\mathrm{H}$ and $\mathrm{M}$ subfamilies contained the greatest numbers of members, while the other five subfamilies possessed less members. The genome structures of these GhTRX genes were investigated by aligning the genomic and cDNA sequences (Fig. 1b). The gene structures of the $\mathrm{O}, \mathrm{F}, \mathrm{Z}, \mathrm{X}$, and Y subfamilies were highly conserved, and the exon-intron number was the same. However, the 14 GhTRX genes in subfamily $\mathrm{H}$ had various exon-intron organizations. For example, Gh_D06G1943,Gh_D09G2058, and Gh_A09G2271 had four exons, Gh_D10G1112 and $G h \_$ A10G1358 had five exons, and the other GhTRX genes in the $\mathrm{H}$ subfamily contained three exons. In addition, the intron lengths in Gh_D10G1112, Gh_A10G1358, and Gh_D06G1943 were longer than those in other members. The length of intron 1 in $G h \_A 05 G 1627$ was the shortest, resulting in variations in gene lengths (Supplementary file $3)$. The genes in the $\mathrm{H}$ subfamily varied from $374 \mathrm{bp}\left(G h_{-}\right.$ $A 05 G 1627)$ to 4975 bp (Gh_A10G1358). The 15 genes in the $\mathrm{M}$ subfamily possessed two exons and one intron, except Gh_D13G1537, which had three exons and two introns. The 15 TRX genes ranged from 576 bp (Gh_D03G1721) to 2,237 bp (Gh_A04G0254). The diversity of the exon-intron structures among the 40 typical TRX genes in cotton indicated differences in expansion and evolution during the polyploidization process.

A protein domain analysis revealed that six different motifs among the 40 typical TRX proteins, with all, except Gh_D10G1930, containing the TRX motif (Fig. 1c). In addition to the TRX domain, these GhTRX proteins contained several other known functional domains, including Thioredoxin_9, AhpC-TSA, Thioredoxin_2, Thioredoxin_7, and TraF.

Phylogenetic and structural analyses of the 46 atypical GhTRX genes revealed that they were divided into nine main subfamilies, PDI11, TSP1, TDX, TRX2, TSP2, 
A

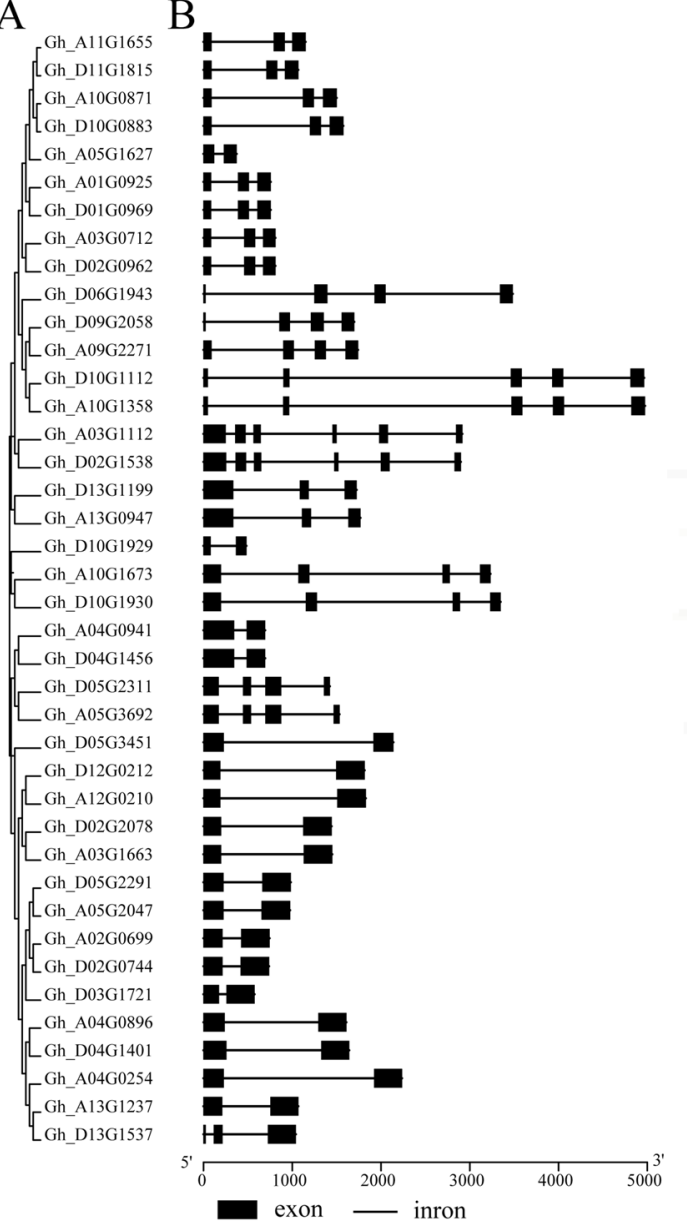

Fig. 1 Phylogenetic relationships, gene structure, and conserved motifs of typical GhTRX gene members. a Phylogenetic tree constructed using the amino acid sequences of 40 typical GhTRX proteins. b The exon-intron structure of typical GhTRX genes. c The characterization of the putative motifs in typical GhTRX proteins. Differently colored boxes represent various types of con-

WCRKC, ACHT, TSP3, and NTRC (Fig. 2a, b). Of the nine subfamilies, the ACHT subfamily contained the greatest number of genes. The GhTRX genes varied from $501 \mathrm{bp}$ (Gh_A06G0134 and Gh_D06G0113) to 7,113 bp (Gh_ A11G1006), whereas the CDS lengths ranged from $270 \mathrm{bp}$ (Gh_A05G3165) to 1,788 bp (Gh_A11G1006) (Supplementary Table S3). Compared with the typical GhTRX genes, the exon-intron organizations of the atypical GhTRX genes were more complex. For example, Gh_A11G1006 contained 16 exons and 15 introns, whereas $G h_{-} A 06 G 0134$ and $G h_{-}$ D06G0113 contained one exon and had no introns (Fig. 2b). A motif analysis of these atypical GhTRX proteins identified 13 domains, and a majority of the proteins harbored one TRX motif (Fig. 2c), indicating that these proteins were highly conserved during the evolution of Gossypium plants. However, proteins in the PDI1 1 and NTRC subfamilies harbored three or four motifs, indicating that their functions

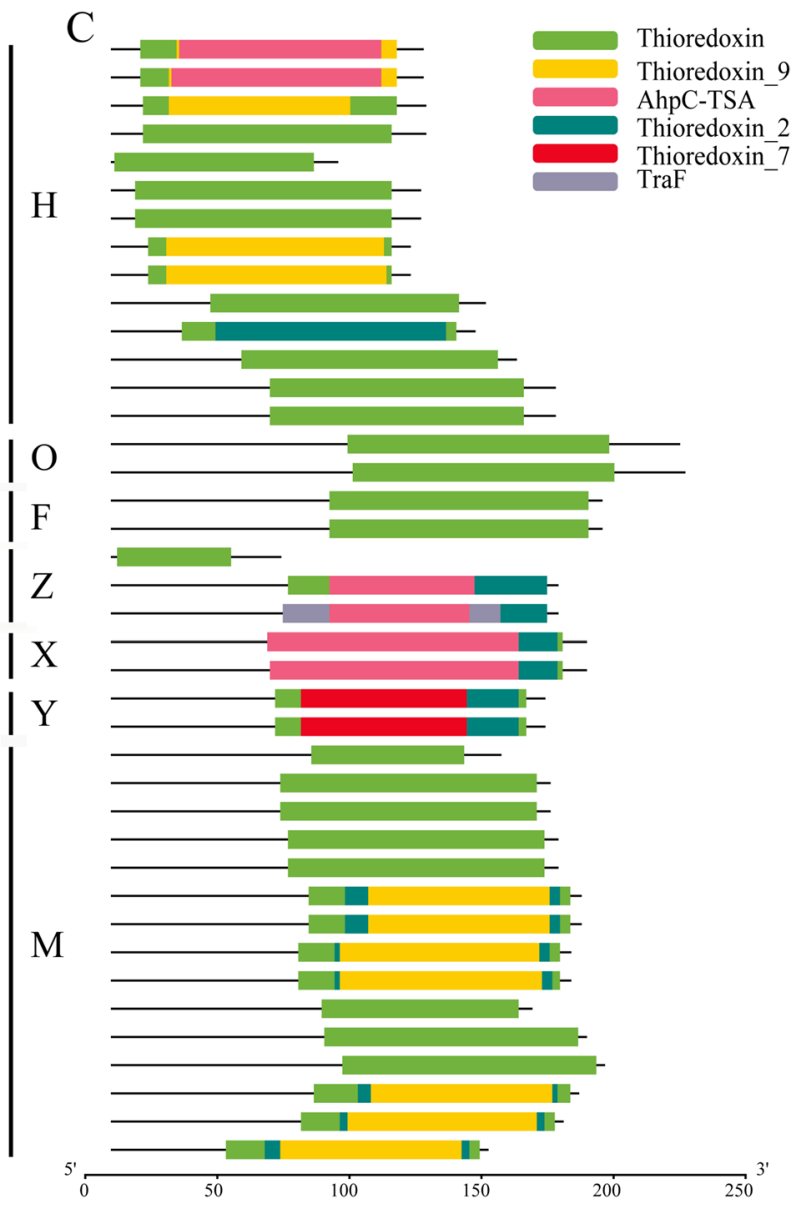

served motifs. Thioredoxin_9 (PF14595), thioredoxin. AhpC-TSA (PF00578), alkyl hydroperoxide reductase (AhpC), and thiol specific antioxidant (TSA). Thioredoxin_2 (PF13098), thioredoxinlike domain. Thioredoxin_7 (PF13899), Thioredoxin-like. traF (PF13728), is a periplasmic protein

differed. Members of the same GhTRX subfamily exhibited similar gene structures and conserved protein domains (Figs. 1 and 2), suggesting that GhTRX gene functions had either been conserved or differentiated during evolution.

\section{Chromosomal Distributions of the GhTRX Genes}

Chromosomal mapping revealed that these 82 GhTRXs were unevenly distributed among the 26 chromosomes (Fig. 3). However, four TRX genes (Gh_A09G2271, Gh_A05G3692, $G h \_A 04 G 1345$, and $\left.G h \_D 03 G 1721\right)$ localized to four scaffolds (Supplementary files 3 and 4). There were 1-7 genes per chromosome: one gene on each of the chromosomes A01, D01, D03, A07, and D07; two genes on chromosomes A02, A09, D08, D09, and A12; three genes on chromosomes A06, D06, A08, A10, and D12; four genes on chromosomes A03, A04, D10, A11, D11, A13, and D13; five genes on 


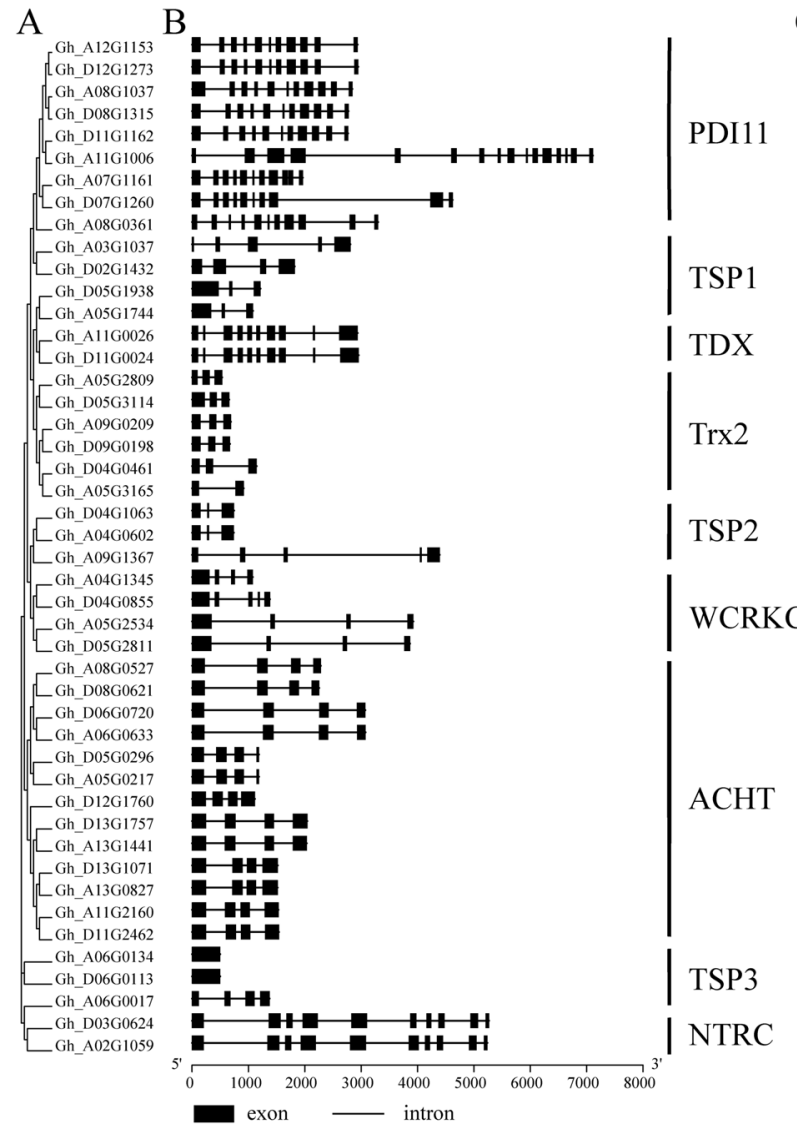

Fig. 2 Phylogenetic clustering, gene structures, and conserved motifs of atypical GhTRX gene members. a Phylogenetic tree constructed using the amino acid sequences of 46 atypical GhTRX proteins. b The exon-intron structures of atypical GhTRX genes. c A characterization of the putative motifs in atypical GhTRX proteins. Differently colored boxes represent various types of conserved motifs. ERp29 (PF07749), Endoplasmic reticulum protein ERp29. OST3 OST6 (PF04756), OST6 family, transporter family. Thioredoxin_2

chromosomes D02 and D04; and seven genes on chromosomes A05 and D05. In total, 40 TRX genes were located on the $\mathrm{A}_{\mathrm{t}}$ subgroup, $42 T R X$ genes were located on the $\mathrm{D}_{\mathrm{t}}$ subgroup. Some GhTRX genes were only distributed in subgroup $\mathrm{A}_{\mathrm{t}}$ or $\mathrm{D}_{\mathrm{t}}$, with no corresponding homoeologous genes found in the other subgroup, such as Gh_A02G1059, Gh_ A03G0712, and Gh_D09G2058. It is possible that individual homoeologous genes in the $A_{t}$ or $D_{t}$ subgroup had undergone translocation or been lost during evolution.

\section{Duplication and Evolutionary Analyses of the GhTRX genes}

The $\mathrm{Ka} / \mathrm{Ks}$ ratio is a measure that explores the mechanisms of gene divergence after duplication. In general, $K a / K s=1$ indicates neutral selection, $K a / K s<1$ indicates purifying selection, and $K a / K s>1$ indicates accelerated evolution with positive selection (Nekrutenko et al. 2002). We calculated 39

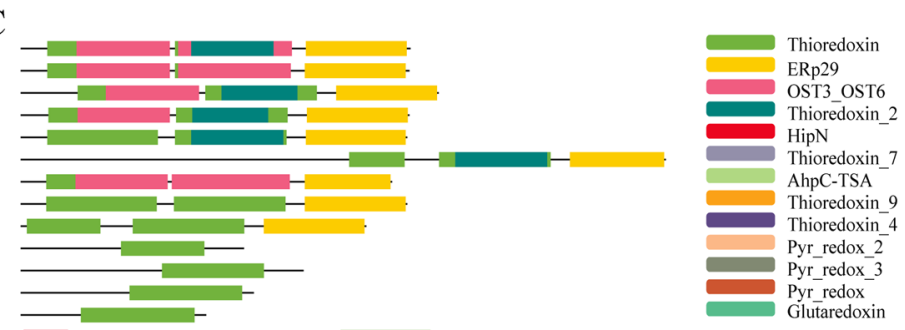

(PF13098), thioredoxin-like domain. HipN (PF18253), Hsp70interacting protein N N-terminal domain. Thioredoxin_7 (PF13899), Thioredoxin-like. AhpC-TSA (PF00578), alkyl hydroperoxide reductase (AhpC) and thiol specific antioxidant (TSA). Thioredoxin_9 (PF14595) and Thioredoxin_4 (PF13462), thioredoxin. Pyr_redox_2 (PF07992) and Pyr_redox_3 (PF13738), pyridine nucleotide-disulphide oxidoreductase. Glutaredoxin (PF00462), Glutaredoxin

duplicated homoeologous pairs in the GhTRX gene family (Supplementary file 5). The Ka/Ks ratios of 33 homoeologous pairs (e.g., Gh_D10G0883/Gh_A10G0871, accounting for $84.62 \%$ ) < 1 , suggesting purifying selection. However, the $\mathrm{Ka} / \mathrm{Ks}$ ratios of six homoeologous gene pairs ( $G h_{-}$ D11G1815/Gh_A11G1655, Gh_D01G0969/Gh_A01G0925, Gh_D02G0962/Gh_A03G0712, Gh_D10G1930/Gh_ A10G1673, Gh_D09G0198/Gh_A09G0209, and Gh_ D05G1938/Gh_A05G1744) were > 1, suggesting positive selection. The positive genes only accounted for $15.38 \%$. Additionally, a collinearity analysis revealed that, among the 86 GhTRX genes, 36 pairs of genes were syntenic between the $A_{t}$ and $D_{t}$ subgenomes (Supplementary Fig. S1).

\section{Expression Profiles of GhTRX Genes in G. hirsutum}

To explore the possible roles of the GhTRX gene family during cotton development, we investigated their 

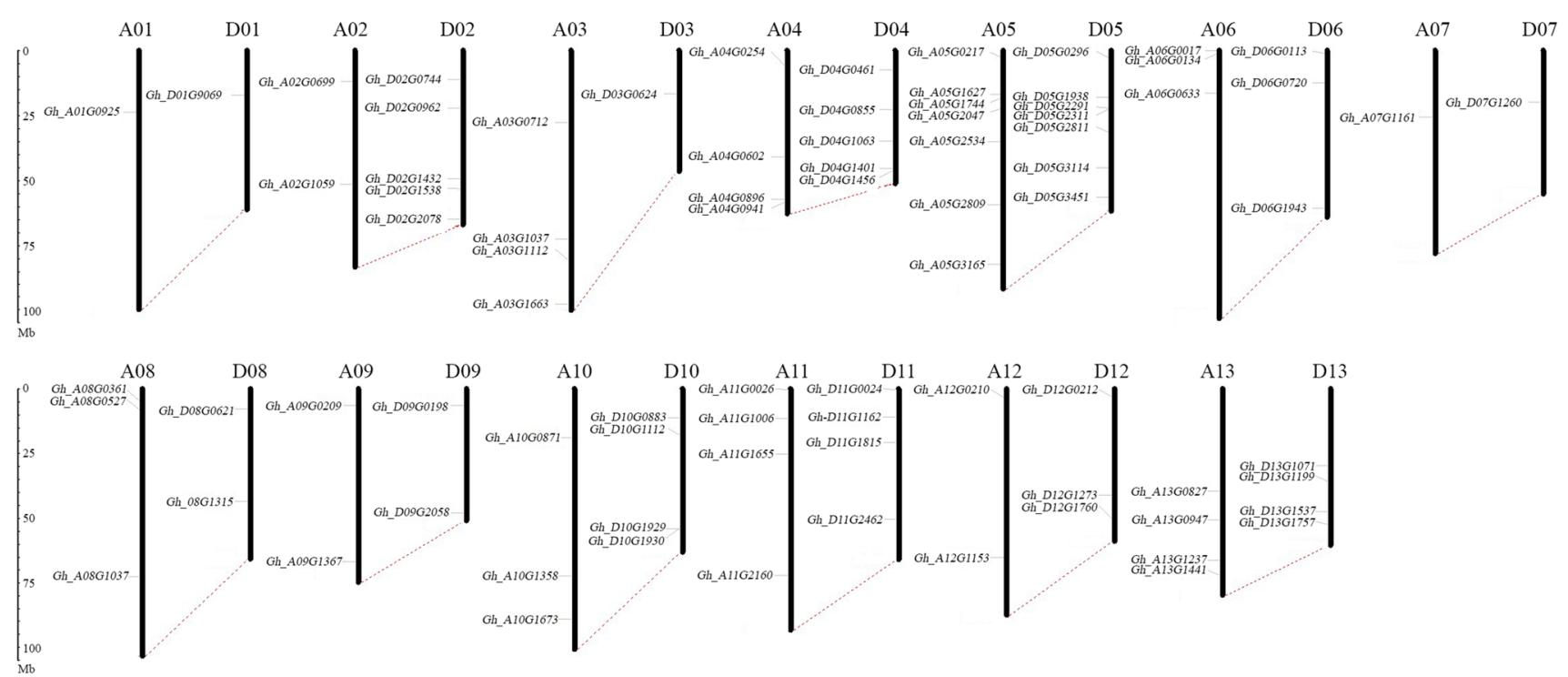

Fig. 3 Chromosomal locations and distributions of the identified GhTRX genes. Dotted lines connect the GhTRX genes present on duplicated chromosomal segments

expression patterns in various organs and during the developmental stages of ovules and fibers using publicly available RNA-Seq data (Hu et al. 2019). The 40 typical GhTRX genes mainly displayed two clusters of expression profiles. One cluster contained eight genes, which were broadly expressed and had high expression levels in all the tested tissues (Fig. 4), suggesting that they play important roles during the growth and development of cotton. The other clusters were categorized into two subclusters. Six GhTRX genes in one subcluster exhibited a ubiquitous expression pattern, with low expression levels in the tested tissues. Most of the typical GhTRXs in the other subcluster were expressed at no or very low levels in the tested tissues, or were only expressed in specific tissues, indicating their specific roles in controlling tissue development.

On the basis of the expression profiles, the 46 atypical GhTRXs were divided into three subclusters (Fig. 5). Among them, subcluster 1 contained six genes, which were highly expressed in all the tested tissues, suggesting their key roles in regulating the growth and development of cotton. Subcluster 2 contained eight genes, which were expressed at relatively high levels in all the tested tissues. Subcluster 3 contained most of the atypical GhTRX genes. The expression levels of these genes were no too very low, or they were only expressed in specific tissues. For example, Gh_A05G0217 and Gh_D060113 were mainly expressed in vegetative organs and floral tissues, while they were not, very lowly, expressed in ovules and fibers. Gh_A05G2534, i.e., GhTRX3-2, was predominantly expressed in stems, leaves, and pistils and presented its highest expression level in leaves. Thus, the GhTRX genes exhibited diverse tissue-specific expression patterns, suggesting that their functions were either conserved or differentiated during evolution.

\section{The Cotton FT Interacted with GhTRXL3-2}

The GhTRXL3-2 was identified through Y2H cDNA library screening assays using GhFT as the bait (data not shown). To confirm the interaction between GhFT and GhTRXL3-2, we first performed a $\mathrm{Y} 2 \mathrm{H}$ experiment. The results demonstrated that GhTRXL3-2 interacted with GhFT in yeast cells (Fig. 6a). We next carried out SLFC assays performed in $N$. benthamiana leaf mesophyll cells and further confirmed these protein interaction results (Fig. 6b).

\section{Overexpression of the GhTRXL3-2 Gene in Arabidopsis Caused Early Flowering}

As the interaction of GhTRXL3-2 and GhFT protein (Fig. 6), we inferred that GhTRXL3-2 functioned in controlling flowering. Therefore, we amplified the open-reading frame cDNA of GhTRXL3-2, an atypical TRX gene, from G. hirsutum cv. 'XLZ 33' in this study using RTPCR. Sequencing confirmed that the amino acid sequence encoded by GhTRXL3-2 gene contains the atypical conserved motif WCRKC shared by plant TRXs. An amino acid sequence alignment revealed that the deduced protein encoded by GhTRXL3-2 is an atypical TRX (Supplementary Fig. S2). Tissue expression analysis, as assessed by qRT-PCR, showed that GhTRXL3-2 was highly expressed in leaves and stems, moderately expressed in flowers, SAM, but hardly expressed in ovules and fibers (Supplementary Fig. S3). The qRT-PCR results were mostly 


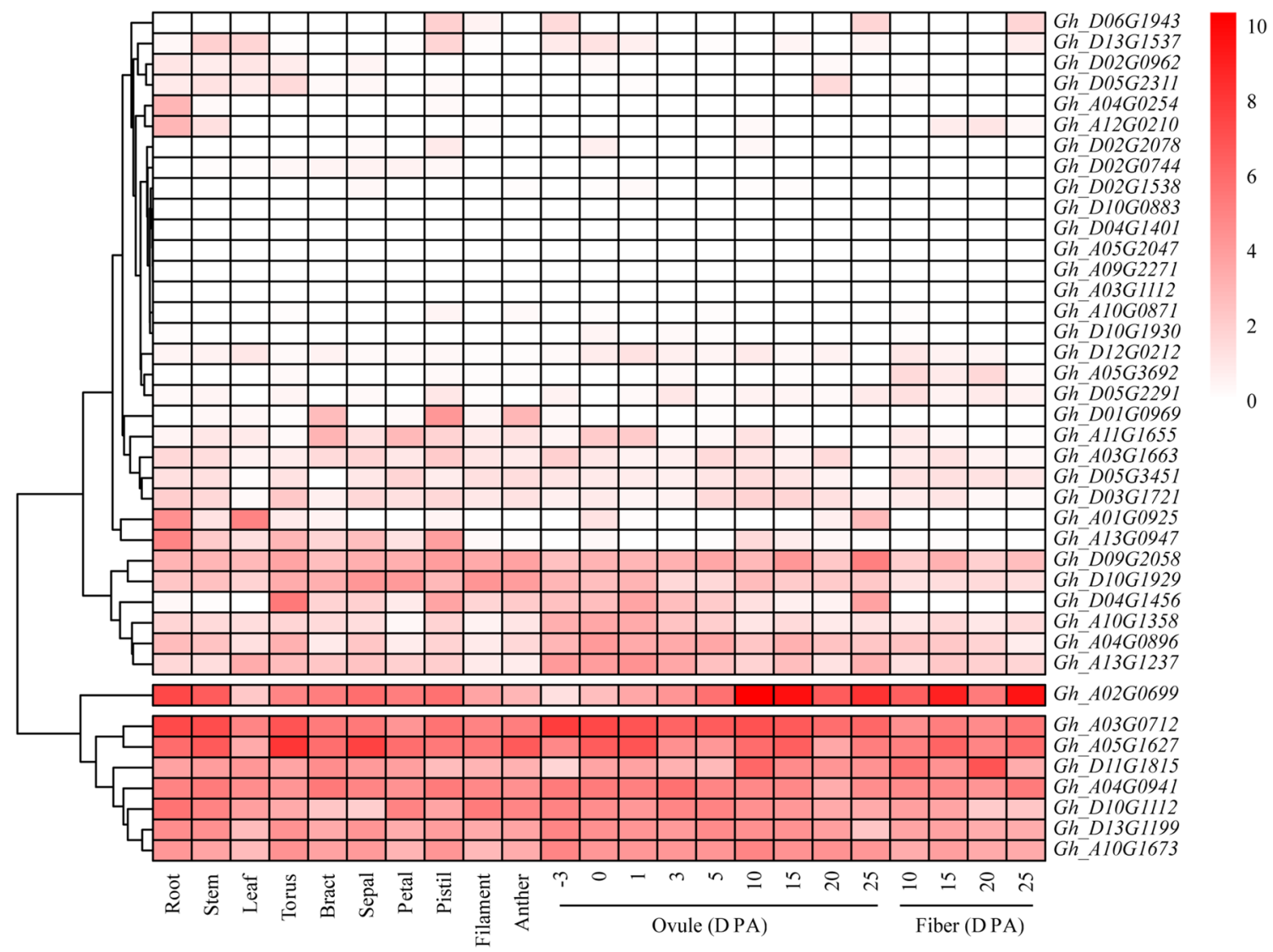

Fig. 4 Expression profiles of typical TRX genes in various tissues. The heat map of the expression levels of 40 typical GhTRX genes in 15 tissues. Fiber-bearing ovules were sampled on $0,1,3$, and 5 days post-anthesis (DPA), and fibers were sampled on 10, 15, 20, and 25 DPA

consistent with the RNA-Seq data (Fig. 5). Because cotton is recalcitrant to regeneration, we initially overexpressed it in Arabidopsis Col-0 plants. As a result, we obtained more than six independent $\mathrm{T}_{3}$ homozygous plants. All the transgenic plants showed the identical early flowering phenotype compared with wild-type (Col-0) plants (Fig. 7a). For example, under LD conditions, the average numbers of days until flowering for lines L21 and L31 were $19.23 \pm 0.25$ and $18.43 \pm 0.16$, respectively, which were significantly less than the $23.83 \pm 0.69$ days observed for Col-0 plants (Fig. 7b). The average numbers of rosette leaves in the lines L21 and L31 were 7.05 \pm 0.19 and $6.96 \pm 0.20$, respectively, which was significantly less than the value of $12.42 \pm 0.45$ found in Col-0 (Fig. 7c). Gene expression, as assessed by qRT-PCR, confirmed that GhTRXL3-2 was overexpressed in the transgenic plants (Fig. 7d). Additionally, greater GhTRXL3-2 expression was observed in the earlier flowering plants than in the transgenic plants not having this phenotype. In addition, two key genes in the flowering pathway, $F T$ and $S U P$ PRESSOR OF OVEREXPRESSION OF CONSTANS 1 (SOC1) (Fornara et al. 2010), were markedly upregulated in transgenic Arabidopsis (Fig. 7e, f).

\section{Silencing of GhTRXL3-2 Delayed Flowering}

To suppress endogenous GhTRXL3-2 expression, we performed a VIGS assay on 2-week-old seedlings having two fully expanded cotyledons in a growth chamber with controlled temperature and humidity. At approximately 11 days post-agroinfiltration, the newly emerging true leaves showed an albino phenotype in plants expose to GhCLA1-expressing agrobacteria (Supplementary Fig. S4), indicating that the VIGS system was functioning. At approximately 2 months post-agroinfiltration, plants infiltrated with the mixture of Agrobacterium cultures containing the pTRV1 and pTRV2 vectors (TRV:00) began to flower, whereas plants infiltrated with the TRV:GhTRXL3-2 flowered later (Fig. 8a, b). qRT-PCR analysis demonstrated that GhTRXL3-2 expression was decreased in the TRV:GhTRVL3-2 lines compared with the TRV:00 lines (Fig. 8c). In addition, GhFT expression levels in the TRV:GhTRVL3-2 lines were significantly lower than that of control (Fig. 8c). 


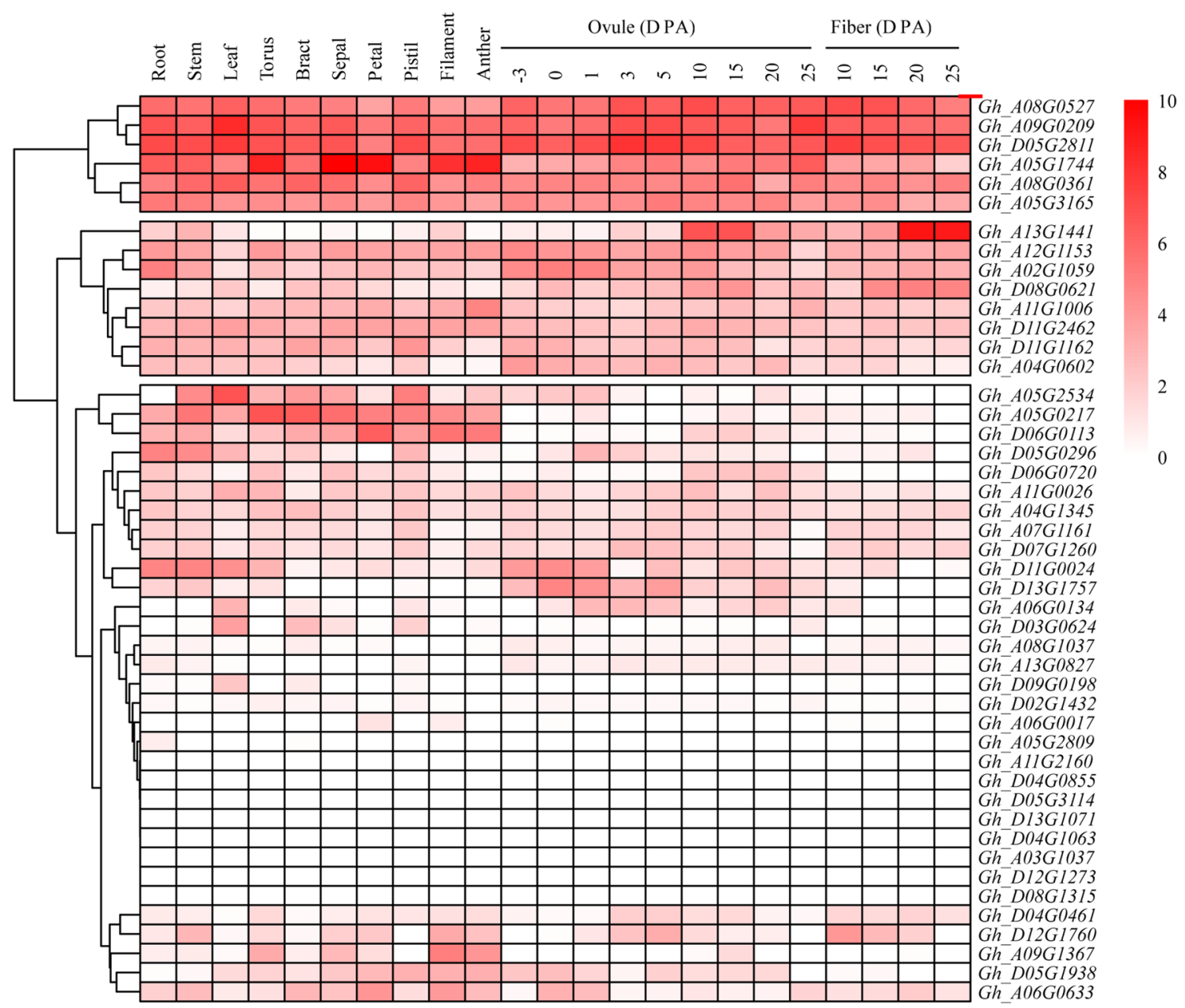

Fig. 5 Expression profiles of atypical TRX genes in various tissues. The heat map of the expression levels of 46 atypical GhTRX genes in 15 tissues. Fiber-bearing ovules were sampled on $0,1,3$, and 5 days post-anthesis (DPA), and fibers were sampled on 10, 15, 20, and 25 DPA

\section{Discussion}

As a small ubiquitous protein found in all organisms, TRX plays important roles in a variety of cellular processes, including transcriptional regulation, metabolism, and signaling. The objectives of this study were to characterize the GhTRX genes, elucidate their expression profiles at different cotton tissues, and select the most appropriate candidate genes for further functional analyses.

\section{Duplication, Functional Conservative, and Divergence of the TRX Genes in Upland Cotton}

The variable state of genome-wide duplication in plants leads to multiple members of most gene families. We found that genome-wide duplication played a crucial role in the expansion of cotton $T R X$ genes, leading to a rapid increase in the number of TRX genes during the evolution of cotton (Supplementary files 3 and 4; Figs. 1 and 2). Similarly, whole-genome- and tandem/segmental duplications resulted in the expansion of the typical TRX gene family in rice (Nuruzzaman et al. 2012). The Ka of most GhTRX genes $(86.4 \%)$ is far less than the $K s$, resulting in $K a$ to $K s$ ratios $<1$ (Supplementary file 5), which indicates that purifying selection is the main type of gene evolution. Among them, six pairs of genes, four typical and two atypical, had $K a / K s>1$, indicating that these $T R X$ genes had been subjected to positive selection. Consequently, we speculated that the GhTRX genes are undergoing purifying selection, which is very important for their functions. Some genes experiencing accelerated evolution to gain new functions.

A genome-wide survey and conserved protein domain analysis identified 86 GhTRX genes, 40 typical and 46 atypical TRXs, in the G. hirsutum genome. In Arabidopsis, at least 20 TRXs have been reported, and most of these proteins are typical TRXs, containing a conserved WC[G/P]PC catalytic site (Meyer et al. 2002). The continued development of biotechnology has led to the discovery of some atypical 
Fig. 6 Interactions among GhTRXL3-2 and GhFT. a Yeast two-hybrid assays of interactions between GhTRXL3-2 and GhFT proteins. b Split firefly luciferase complementation assays showing interactions between GhTRXL3-2 and GhFT protein in N. benthamiana leaf epidermal cells. The pseudocolor bar in image shows the range of luminescence intensity. $\mathrm{Bar}=50 \mu \mathrm{m}$
A

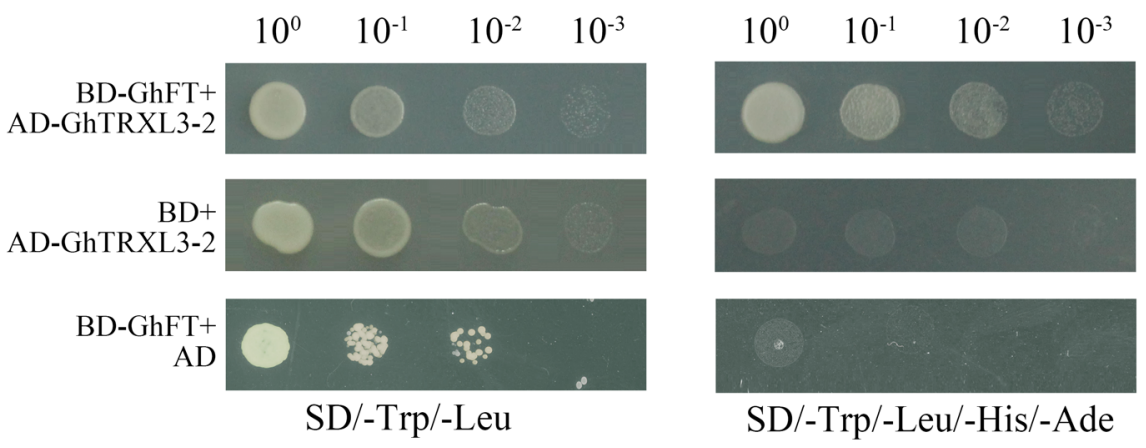

B

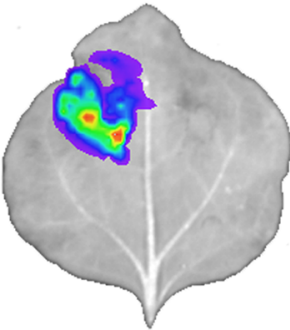

GhFT-nLUC

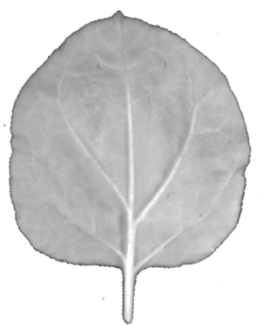

GhFT-nLUC cI $\stackrel{+}{\mathrm{U}} \mathrm{C}$

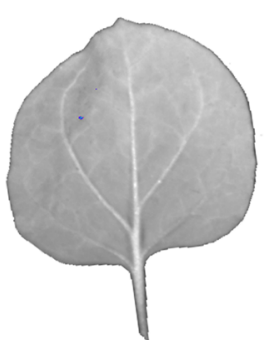

$\mathrm{nLUC}_{+}$

cLUC-GhTRXL3-2

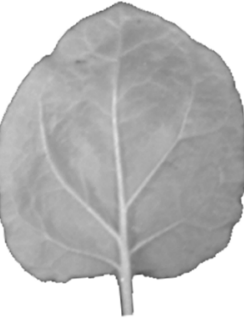

nLUC

cLü

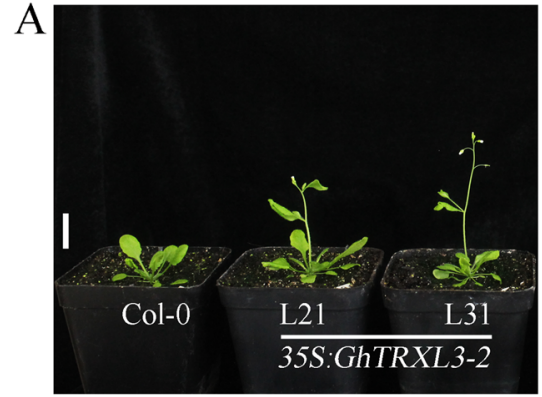

D

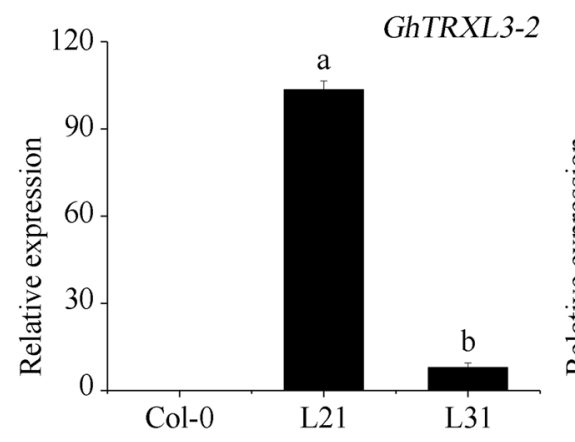

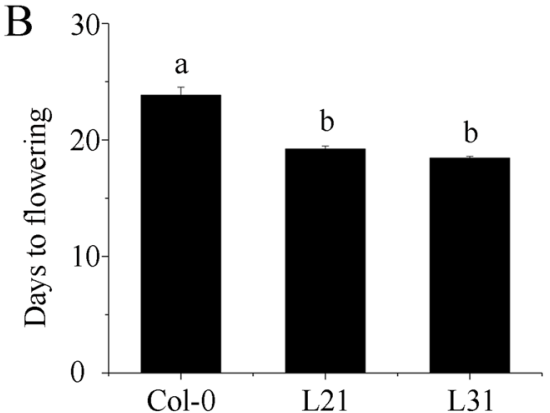

E

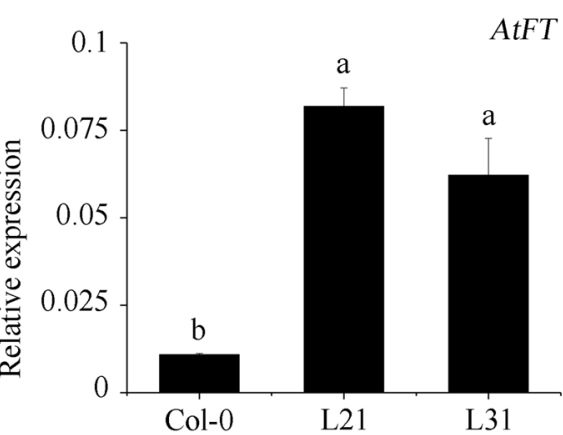

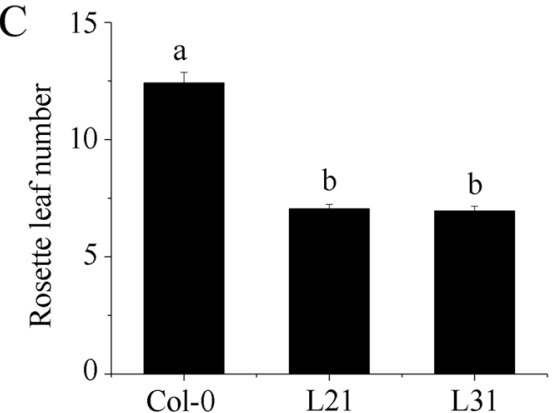

F

AtSOC1

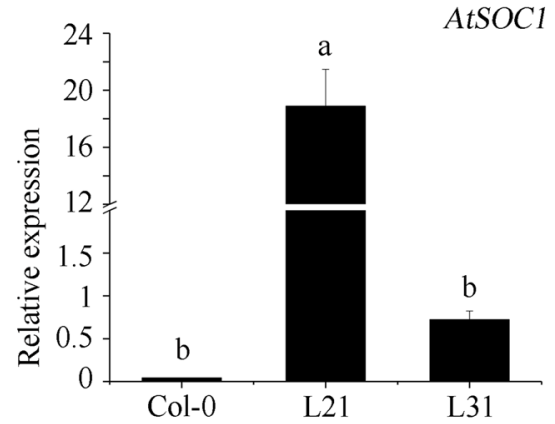

Fig. 7 Ectopic overexpression of GhTRXL3-2 in Arabidopsis caused early flowering. a Phenotype of 18-day-old Arabidopsis Col-0 and 35S:GhTRXL3-2 transgenic plants grown under long-day (16-h light/8-h dark) conditions in a growth chamber. Scale bar, $2 \mathrm{~cm}$. b The days to flowering as determined by the day floral buds became visible. c Flowering time in representative lines as determined by number of rosette leaves at flowering. d Expression analysis of GhTRXL3-2 in Col-0 and transgenic Arabidopsis plants as assessed

by qRT-PCR. e, $\mathbf{f}$ qRT-PCR analysis of AtFT and AtSOC1 expression levels in different transgenic Arabidopsis lines. Arabidopsis ACT2 (At3g18780) was used as the internal reference gene. AtFT, At1g65480; AtSOC1, At2g45660. Values are means \pm SDs $(n=3)$. Different lowercase letters represent statistically significant differences among Col-0 and the 35S:GhTRXL3-2 transgenic lines as determined by a one-way ANOVA $(P<0.05$, Duncan's multiple range test) 

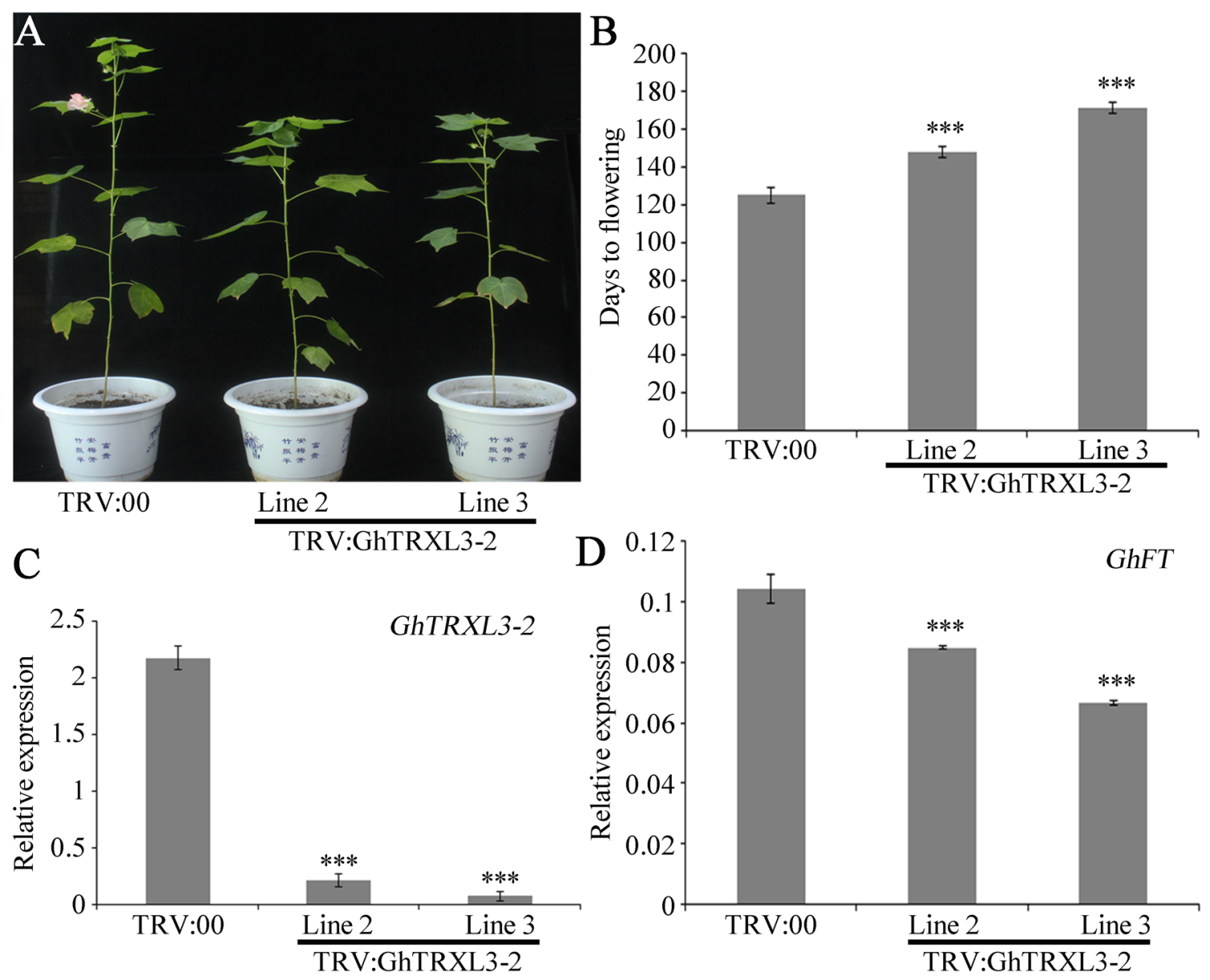

Fig. 8 Agrobacterium-mediated virus-induced gene silencing (VIGS) in cotton. a Phenotypic comparison of the empty vector control TRV:00 and the TRV:GhTRXL3-2 silencing lines. b The days to flowering as determined by the day the first flower opened.

TRXs, such as Trx-like and Trx-lilium, which have different active site sequences (Cain et al. 2009; Dangoor et al. 2009). The 40 typical GhTRXs were distributed in $\mathrm{H}, \mathrm{O}, \mathrm{F}$, $\mathrm{Z}, \mathrm{X}, \mathrm{Y}$, and M subgroups, and most of which have WCGPC active sites (Fig. 1). The 46 atypical GhTRXs comprised ACHT, TDX, Trx2, WCRKC, PDI11, NTRC, and TSP subgroups (Fig. 2). These atypical subfamilies contained different numbers of $T R X$ genes and different active sites, which might be closely correlated with their various functions. The increased number of genes in some subfamilies may be an evolutionary result of adapting to environmental changes, while gene duplication is species-specific.

\section{The Atypical TRX Member, GhTRXL3-2, Promotes Plant Flowering}

Reactive oxygen species and related reduction/oxidation involving TRXs fulfill crucial functions in flower development and flowering (Schippers et al. 2016). Because of

The expression levels of GhTRXL3-2 (c) and GhFT (d) in nonsilenced and silenced plants detected by semi-quantitative RT-PCR. Unpaired $\mathrm{t}$ tests were performed to determine statistical significance at “***" $P<0.001$

the interaction of GhFT and GhTRXL3-2 in vivo and vitro (Fig. 6), we speculated that GhTRXL3-2 plays a role in flowering. In this study, the overexpression of GhTRXL3-2 increased the transcription levels of genes related to flowering, such as $F T$ and $S O C 1$, in A. thaliana and led to early flowering (Fig. 7). Moreover, the silencing of GhTRXL3-2 reduced the expression level of GhFT and delayed flowering (Fig. 8). These results confirmed that GhTRXL3-2 functions in cotton flowering and development in cotton. However, whether GhTRXL3-2' function in flowering involves another flowering pathway requires further study. Research on cotton TRXs might be of great significance for the functional genomics and modern breeding of cotton. 


\section{Conclusion}

A genome-wide investigation of TRX family genes identified $86 T R X$ genes in upland cotton. On the basis of the presence of the TRX domain, the identified TRX genes were sub-grouped into two classes, typical (40) and atypical (46). The gene structures, conserved motifs, chromosomal locations, evolutionary relationships, and expression patterns were comprehensively examined in this study. In addition, an RNA-Seq analysis revealed that, during vegetative and reproductive development, the difference in abundances and the distinct expression patterns of these $T R X$ genes suggested varied functions. The overexpression of GhTRXL3-2 in Arabidopsis promoted flowering, while the silencing of GhTRXL3 in cotton resulted in delayed flowering, revealing its crucial role in flowering regulation. Our results provided a foundation for future studies into new applications in a range of fields and a better understanding of GhTRX functions during cotton growth and development.

Supplementary Information The online version contains supplementary material available at https://doi.org/10.1007/s12374-021-09318-1.

Author contributions $\mathrm{XH}$ conceived and organized the experiments. HL performed Y2H, SFLC, qRT-PCR, and genetic transformation experiments. YL carried out gene family analysis. XH wrote the manuscript. All authors have read and approved the final manuscript.

Funding This study was supported by grants from the National Natural Science Foundation of China $(31860393,31360366)$, and the Talent Introduction Start-up Fund Project of Anhui Science and Technology University (NXYJ202001).

\section{Declarations}

Conflicts of Interest The authors declare no conflict of interest.

Open Access This article is licensed under a Creative Commons Attribution 4.0 International License, which permits use, sharing, adaptation, distribution and reproduction in any medium or format, as long as you give appropriate credit to the original author(s) and the source, provide a link to the Creative Commons licence, and indicate if changes were made. The images or other third party material in this article are included in the article's Creative Commons licence, unless indicated otherwise in a credit line to the material. If material is not included in the article's Creative Commons licence and your intended use is not permitted by statutory regulation or exceeds the permitted use, you will need to obtain permission directly from the copyright holder. To view a copy of this licence, visit http://creativecommons.org/licenses/by/4.0/.

\section{References}

Bailey TL, Johnson J, Grant CE, Noble WS (2015) The MEME suite. Nucleic Acids Res 43(W1):W39-49
Cain P, Hall M, Schröder WP, Kieselbach T, Robinson C (2009) A novel extended family of stromal thioredoxins. Plant Mol Biol 70(3):273-281

Chen C, Chen H, Zhang Y, Thomas HR, Frank MH, He Y, Xia R (2020) TBtools: An Integrative Toolkit Developed for Interactive Analyses of Big Biological Data. Mol Plant 13(8):1194-1202

Chibani K, Tarrago L, Gualberto JM, Wingsle G, Rey P, Jacquot JP, Rouhier N (2012) Atypical thioredoxins in poplar: the glutathione-dependent thioredoxin-like 2.1 supports the activity of target enzymes possessing a single redox active cysteine. Plant Physiol 159(2):592-605

Clough SJ, Bent AF (1998) Floral dip: a simplified method for Agrobacterium-mediated transformation of Arabidopsis thaliana. Plant J 16(6):735-743

Dangoor I, Peled-Zehavi H, Levitan A, Pasand O, Danon A (2009) A small family of chloroplast atypical thioredoxins. Plant Physiol 149(3):1240-1250

Delorme-Hinoux V, Bangash SA, Meyer AJ, Reichheld JP (2016) Nuclear thiol redox systems in plants. Plant Sci 243:84-95

Fornara F, de Montaigu A, Coupland G (2010) SnapShot: control of flowering in Arabidopsis. Cell 141(3):550

Gao X, Britt RC Jr, Shan L, He P (2011) Agrobacterium-mediated virus-induced gene silencing assay in cotton. J Vis Exp 54:2938

Gelhaye E, Rouhier N, Navrot N, Jacquot JP (2005) The plant thioredoxin system. Cell Mol Life Sci 62(1):24-35

Guo D, Li C, Dong R, Li X, Xiao X, Huang X (2015) Molecular cloning and functional analysis of the FLOWERING LOCUS T (FT) homolog GhFT1 from Gossypium hirsutum. J Integr Plant Biol 57(6):522-533

Heide H, Kalisz HM, Follmann H (2004) The oxygen evolving enhancer protein 1 (OEE) of photosystem II in green algae exhibits thioredoxin activity. J Plant Physiol 161(2):139-149

Hu B, Jin J, Guo AY, Zhang H, Luo J, Gao G (2015) GSDS 2.0: an upgraded gene feature visualization server. Bioinformatics 31(8):1296-1297

Hu Y, Chen J, Fang L, Zhang Z, Ma W, Niu Y, Ju L, Deng J, Zhao T, Lian J, Baruch K, Fang D, Liu X, Ruan YL, Rahman MU, Han J, Wang K, Wang Q, Wu H, Mei G, Zang Y, Han Z, Xu C, Shen W, Yang D, Si Z, Dai F, Zou L, Huang F, Bai Y, Zhang Y, Brodt A, Ben-Hamo H, Zhu X, Zhou B, Guan X, Zhu S, Chen X, Zhang T (2019) Gossypium barbadense and Gossypium hirsutum genomes provide insights into the origin and evolution of allotetraploid cotton. Nat Genet 51(4):739-748

Huang G, Wu Z, Percy RG, Bai M, Li Y, Frelichowski JE, Hu J, Wang K, Yu JZ, Zhu Y (2020) Genome sequence of Gossypium herbaceum and genome updates of Gossypium arboreum and Gossypium hirsutum provide insights into cotton A-genome evolution. Nat Genet 52(5):516-524

Krzywinski M, Schein J, Birol I, Connors J, Gascoyne R, Horsman D, Jones SJ, Marra MA (2009) Circos: an information aesthetic for comparative genomics. Genome Res 19(9):1639-1645

Kumar S, Stecher G, Tamura K (2016) MEGA7: Molecular evolutionary genetics analysis version 7.0 for bigger datasets. Mol Biol Evol 33(7):1870-1874

Li C, Zhang Y, Zhang K, Guo D, Cui B, Wang X, Huang X (2015) Promoting flowering, lateral shoot outgrowth, leaf development, and flower abscission in tobacco plants overexpressing cotton FLOWERING LOCUS T (FT)-like gene GhFT1. Front Plant Sci 6:454

Meyer Y, Vignols F, Reichheld JP (2002) Classification of plant thioredoxins by sequence similarity and intron position. Methods Enzymol 347:394-402

Meyer Y, Siala W, Bashandy T, Riondet C, Vignols F, Reichheld JP (2008) Glutaredoxins and thioredoxins in plants. Biochim Biophys Acta 1783(4):589-600 
Meyer Y, Buchanan BB, Vignols F, Reichheld JP (2009) Thioredoxins and glutaredoxins: unifying elements in redox biology. Annu Rev Genet 43:335-367

Meyer Y, Belin C, Delorme-Hinoux V, Reichheld JP, Riondet C (2012) Thioredoxin and glutaredoxin systems in plants: molecular mechanisms, crosstalks, and functional significance. Antioxid Redox Signal 17(8):1124-1160

Nekrutenko A, Makova KD, Li WH (2002) The K(A)/K(S) ratio test for assessing the protein-coding potential of genomic regions: an empirical and simulation study. Genome Res 12(1):198-202

Nuruzzaman M, Sharoni AM, Satoh K, Al-Shammari T, Shimizu T, Sasaya T, Omura T, Kikuchi S (2012) The thioredoxin gene family in rice: genome-wide identification and expression profiling under different biotic and abiotic treatments. Biochem Biophys Res Commun 423(2):417-423

Potters G, Horemans N, Jansen MA (2010) The cellular redox state in plant stress biology - a charging concept. Plant Physiol Biochem 48(5):292-300

Schippers JH, Foyer CH, van Dongen JT (2016) Redox regulation in shoot growth, SAM maintenance and flowering. Curr Opin Plant Biol 29:121-128

Shi YH, Zhu SW, Mao XZ, Feng JX, Qin YM, Zhang L, Cheng J, Wei LP, Wang ZY, Zhu YX (2006) Transcriptome profiling, molecular biological, and physiological studies reveal a major role for ethylene in cotton fiber cell elongation. Plant Cell 18(3):651-664

Si Z, Liu H, Zhu J, Chen J, Wang Q, Fang L, Gao F, Tian Y, Chen Y, Chang L, Liu B, Han Z, Zhou B, Hu Y, Huang X (2018)
Zhang T (2018) Mutation of SELF-PRUNING homologs in cotton promotes short-branching plant architecture. J Exp Bot 69(10):2543-2553

Taoka K, Ohki I, Tsuji H, Furuita K, Hayashi K, Yanase T, Yamaguchi M, Nakashima C, Purwestri YA, Tamaki S, Ogaki Y, Shimada C, Nakagawa A, Kojima C, Shimamoto K (2011) 14-3-3 proteins act as intracellular receptors for rice $\mathrm{Hd} 3 \mathrm{a}$ florigen. Nature 476(7360):332-335

Trapnell C, Roberts A, Goff L, Pertea G, Kim D, Kelley DR, Pimentel H, Salzberg SL, Rinn JL, Pachter L (2012) Differential gene and transcript expression analysis of RNA-seq experiments with TopHat and Cufflinks. Nat Protoc 7(3):562-578

Wang Y, Tang H, Debarry JD, Tan X, Li J, Wang X, Lee TH, Jin H, Marler B, Guo H, Kissinger JC, Paterson AH (2012) MCScanX: a toolkit for detection and evolutionary analysis of gene synteny and collinearity. Nucleic Acids Res 40(7):e49

Zhang T, Hu Y, Jiang W, Fang L, Guan X, Chen J, Zhang J, Saski CA, Scheffler BE, Stelly DM, Hulse-Kemp AM, Wan Q, Liu B, Liu C, Wang S, Pan M, Wang Y, Wang D, Ye W, Chang L, Zhang W, Song Q, Kirkbride RC, Chen X, Dennis E, Llewellyn DJ, Peterson DG, Thaxton P, Jones DC, Wang Q, Xu X, Zhang H, Wu H, Zhou L, Mei G, Chen S, Tian Y, Xiang D, Li X, Ding J, Zuo Q, Tao L, Liu Y, Li J, Lin Y, Hui Y, Cao Z, Cai C, Zhu X, Jiang Z, Zhou B, Guo W, Li R, Chen ZJ (2015) Sequencing of allotetraploid cotton (Gossypium hirsutum L. acc. TM-1) provides a resource for fiber improvement. Nat Biotechnol 33(5):531-537 